\title{
A Combination of the Aerosolized PPAR-y Agonist Pioglitazone and a Synthetic Surfactant Protein B Peptide Mimic Prevents Hyperoxia-Induced Neonatal Lung Injury in Rats
}

\author{
Reiko Sakurai Cindy Lee Humphrey Shen Alan J. Waring Frans J. Walther \\ Virender K. Rehan \\ Department of Pediatrics, David Geffen School of Medicine, University of California Los Angeles, Torrance, CA, USA; \\ Los Angeles Biomedical Research Institute at Harbor-UCLA Medical Center, Torrance, CA, USA
}

\section{Keywords \\ Bronchopulmonary dysplasia $\cdot$ Chronic lung disease $\cdot$ Lung maturation - Prematurity $\cdot$ Respiratory distress syndrome}

\begin{abstract}
Background: Despite improvements in perinatal care, bronchopulmonary dysplasia (BPD) in extremely premature infants has not decreased. Postnatal surfactant therapy provides symptomatic relief from respiratory distress syndrome, but does not translate into a reduction in BPD. Therefore, the search for effective interventions to prevent BPD continues. Objectives: Since PPAR- $\gamma$ agonists have been demonstrated to promote neonatal lung maturation and injury repair, we hypothesized that a formulation of a PPAR- $\gamma$ agonist, pioglitazone (PGZ) and a synthetic lung surfactant (a surfactant protein $B$ peptide mimic, B-YL) combined would stimulate lung maturation and block hyperoxia-induced neonatal lung injury more effectively than either modality alone. Methods: One-day-old Sprague-Dawley rat pups were administered PGZ + B-YL via nebulization every $24 \mathrm{~h}$ for up to $72 \mathrm{~h}$. The pups were exposed to either 21 or $95 \% \mathrm{O}_{2}$, and then sacrificed. Their lungs were examined for markers of lung maturation (levels of PPAR- $\gamma$, SP-C and choline-phosphate
\end{abstract}

cytidylyltransferase $[\mathrm{CCT}-\mathrm{a}]$ and $\left[{ }^{3} \mathrm{H}\right]$ triolein uptake) and injury repair (bronchoalveolar lavage cell count and protein content, and levels of LEF-1, fibronectin, ALK5, and $\beta$-catenin) by Western blot analysis. Results: Markers of alveolar epithe$\mathrm{lial} /$ mesenchymal maturation (PPAR- $\gamma, \mathrm{SP}-\mathrm{C}, \mathrm{CCT}-\alpha$, and triolein uptake) increased significantly in the PGZ + B-YL group, more than with either drug alone. Similarly, markers of hyperoxia-induced lung injury were blocked effectively with PGZ + B-YL treatment. Conclusions: Nebulized PPAR- $\gamma$ agonist PGZ with a synthetic lung surfactant accelerates lung maturation and prevents neonatal hyperoxia-induced lung injury more than either modality alone, with the potential to provide more effective prevention of BPD.

(c) 2018 S. Karger AG, Basel

\section{Introduction}

Despite improvements in perinatal care, bronchopulmonary dysplasia (BPD) in extremely premature infants has not decreased $[1,2]$. The postnatal use of surfactant provides symptomatic relief from respiratory distress syndrome (RDS), but it does not translate into a reduction in BPD. This is not surprising, since the administra-

\section{KARGER}

(c) 2018 S. Karger AG, Basel

E-Mail karger@karger.com

www.karger.com/neo
Virender K. Rehan, MD 
tion of surfactant alone does not address the fundamental issue in RDS, i.e., lung immaturity [3, 4]. In contrast, PPAR- $\gamma$ agonists recapitulate the natural cellular and molecular pathways that determine normal lung structure, function, and homeostasis. In multiple in vitro and in vivo experimental models of neonatal lung injury, PPAR- $\gamma$ agonists, administered either parenterally [5] or locally [6], enhance lung maturation and prevent neonatal lung injury [5-7]. We reasoned that combining lung surfactant with a PPAR- $\gamma$ agonist would mean they complement each other's benefits, and that the net effect on lung injury reduction would be greater than with either intervention alone. Therefore, we hypothesized that a combined PPAR- $\gamma$ agonist and surfactant formulation effectively blocks neonatal lung injury and that this effect is greater than with either modality alone.

We used a new and innovative surfactant protein $B$ (SP-B) peptide mimic, B-YL, developed for aerosolized delivery specifically in a hyperoxic environment. Using the $\mathrm{N}$-terminal (residues 1-25) and C-terminal (residues 63-78) $\alpha$-helical sequences of native SP-B, connected with a short $\beta$-sheet loop [8], we replaced the cysteine and methionine residues with tyrosine and leucine, respectively, to minimize inactivation by oxidative stress. Based on homology templating of the linear amino acid sequence (NH2-FPIPLPYYWLYRALIKRIQALIPKGGRL LPQLVYRLVLRYS-COOH), the 3-dimensional structure of the SP-B peptide mimic B-YL is shown in Figure 1. As for the choice of PPAR- $\gamma$ agonist, due to the proved superior safety and efficacy of nebulized pioglitazone (PGZ) in blocking hyperoxia-induced neonatal lung injury [5], this was combined with B-YL surfactant. Using a neonatal rat model, we planned to administer a highly effective, stable, and oxidation-resistant synthetic lung surfactant, B-YL, and simultaneously enhance lung maturation via PGZ to prevent hyperoxia-induced neonatal lung injury.

\section{Materials and Methods}

\section{Synthetic Surfactant}

B-YL peptide was synthesized on a Symphony multiple peptide synthesizer (Protein Technologies, Tucson, AZ, USA) using a Fast$\mathrm{Moc}^{\mathrm{TM}}$ protocol on an $\mathrm{H}-\mathrm{Ser}(\mathrm{OtBu})-\mathrm{HMPB}$ NovaPEG resin, as described previously $[8,9]$. Purified B-YL peptide was freeze-dried and its mass was confirmed by MALDI-TOF mass spectrometry. B-YL surfactant was formulated to contain $35 \mathrm{mg} / \mathrm{mL}$ of $5: 3: 2$ DPPC:POPC:POPG combined with 3.0\% (by wt) of B-YL. The synthetic surfactant components were combined in organic solvent (e.g., chloroform for lipids and TFE for peptide), dried under nitrogen, exposed to house vacuum to remove residual solvent, resus-

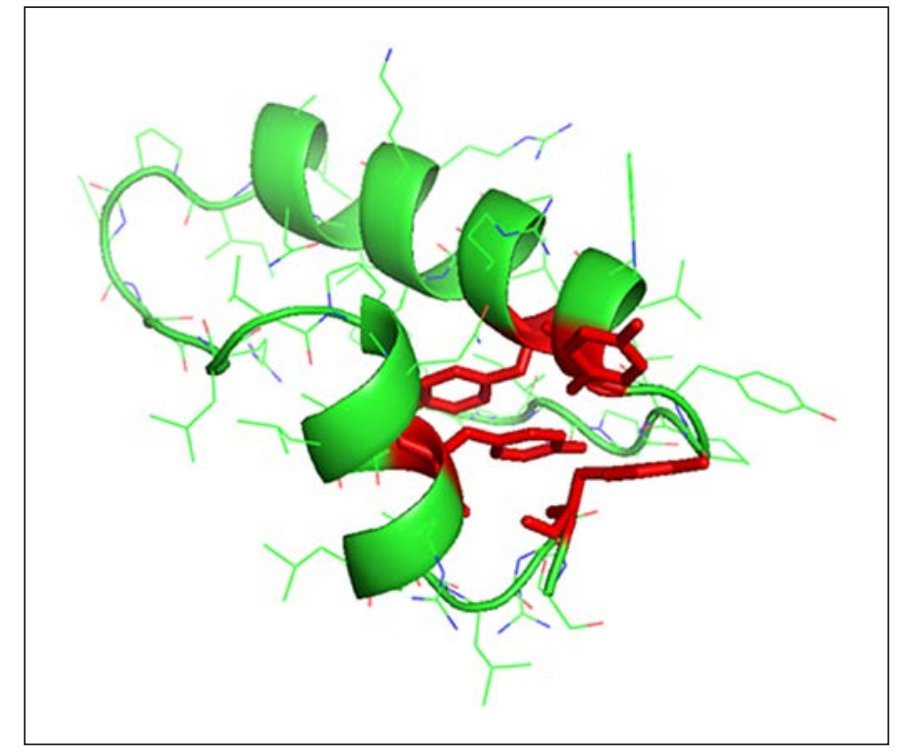

Fig. 1. Predicted molecular model of B-YL derived from the primary amino acid sequence using the homology templating program I-TASSER [26]. The secondary structure of the SP-B peptide mimic B-YL is that of a helix hairpin, with the helical elements (green ribbons) connected by a bend domain (green tube). The helix hairpin structure is stabilized by the interaction of the tyrosine side-chains (in red) at the $\mathrm{N}$ - and $\mathrm{C}$-terminal domains (that emulate the disulfide interactions) and the Saposin protein-fold of the native SP-B sequence.

pended by hand-vortexing in $0.15 \mathrm{M} \mathrm{NaCl}$ adjusted to $\mathrm{pH} 7.0$, heated to $65^{\circ} \mathrm{C}$ intermittently for $30 \mathrm{~min}$, and refrigerated for $>12 \mathrm{~h}$ prior to use. PGZ was mixed into the surfactant as an ethanol suspension ( $4 \mathrm{mg} / \mathrm{mL}$ pure ethanol), followed by removal of the ethanol by evaporation. The surface tension-lowering ability of surfactant preparations was assessed under dynamic compression at $37^{\circ} \mathrm{C}$ by captive bubble surfactometry [8]. We routinely analyzed surfactant samples of $1 \mu \mathrm{L}$ (35 mg phospholipids/mL) in approximately $1.5 \mathrm{~mL}$ of Goerke buffer with 10\% sucrose (approx. 25 $\mu \mathrm{g}$ surfactant/ $\mathrm{mL}$ ) in the captive bubble surfactometer, and performed all measurements in quadruplicate. Lipids only were used as a negative control and the clinical surfactant Infasurf ${ }^{\circledR}$ as a positive control.

Animal Protocol and Study Design

All studies were approved by the Institutional Review Board and conducted in accordance with the NIH Guide for the Care and Use of Laboratory Animals. Briefly, following our previously described protocol, 1-day-old breastfeeding (ad libitum) rat pups were exposed to either normoxia $\left(21 \% \mathrm{O}_{2}\right)$ or hyperoxia $\left(95 \% \mathrm{O}_{2}\right)$ for $72 \mathrm{~h}[6,7]$. Hyperoxia-exposed animals were administered diluent, B-YL (100 mg/kg body weight [BW]), PGZ (1 mg/kg), or PGZ $(1 \mathrm{mg} / \mathrm{kg})+\mathrm{B}-\mathrm{YL}(100 \mathrm{mg} / \mathrm{kg})$ in a $1-\mathrm{mL}$ volume via nebulization over 30 min every $24 \mathrm{~h}$ for up to $72 \mathrm{~h}$. Aerosolization of B-YL and PGZ was achieved with a vibrating mesh nebulizer (Aeroneb Pro ${ }^{\circledR}$ nebulizer, Aerogen Inc., Mountain View, CA, USA) $[9,10]$, which aerosolizes liquid formulations without affecting a compound's composition and concentration. This small-volume (10 


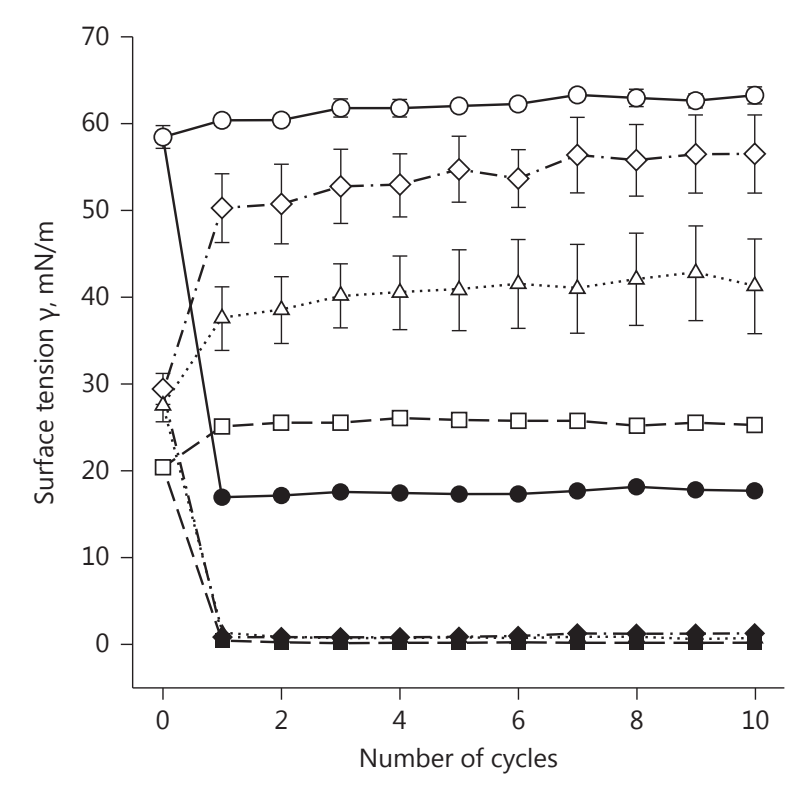

Fig. 2. Comparison of B-YL, PGZ + B-YL, lipids only, and Infasurf on surface tension reduction activity. Surface activity of the B-YL $(100 \mathrm{mg} / \mathrm{kg})$ was measured with captive bubble surfactometry in the presence and absence of PGZ (1 mg/kg), and then compared with a clinical surfactant (Infasurf) as a positive control and with lipids only (DPPC:POPC:POPG 5:3:2 wt:wt:wt) as a negative control. Minimum surface tension values are indicated by black symbols and maximum values by white symbols. Infasurf, squares; B-YL, triangles; B-YL + PGZ, diamonds; lipids, circles. Minimum surface tension values of $\mathrm{B}-\mathrm{YL} \pm \mathrm{PGZ}$ were similar to those of Infasurf (mean \pm SEM of $n=4-5$ ).

$\mathrm{mL}$ maximum), median-diameter nebulizer delivers a low-velocity aerosol with a particle size $<3.0 \mu \mathrm{m}$ and a flow rate $>0.1 \mathrm{~mL} / \mathrm{min}$, is FDA-approved for human use, and does not need a gas source [11]. Control (normoxia group) pups were administered the diluent similarly via nebulization.

All pups were killed at $72 \mathrm{~h}$, and lungs were either snap-frozen for Western blot analysis of markers of lung homeostasis (SP-C and choline-phosphate cytidylyltransferase [CCT]- $\alpha$ levels) and injury repair (LEF-1 and fibronectin levels) or cultured as explants to determine the rate of $\left[{ }^{3} \mathrm{H}\right]$ triolein uptake, a functional marker of surfactant phospholipid synthesis. A separate set of animals was used for bronchoalveolar lavage fluid (BALF) collection. Retrieved BALF was centrifuged at $400 \mathrm{~g}$ for $10 \mathrm{~min}$. Sediments were analyzed for total cell counts and supernatants were snap-frozen in liquid nitrogen for the subsequent analysis of inflammatory markers, such as interleukin (IL)- 6 and interferon (IFN)- $\gamma$ (ELISA kits from R\&D Systems).

\section{Western Blot Analysis}

Following previously described methods $[6,7,12]$, Western blot analysis was performed on lung tissue to determine the protein levels of markers of alveolar epithelial (SP-C and CCT- $\alpha$ ) and alveolar mesenchymal (PPAR- $\gamma$ ) differentiation and injury re- pair (LEF-1 and fibronectin). Primary antibodies used included: PPAR- $\gamma$ (1:500), SP-C (1:250), CCT- $\alpha$ (1:200), LEF-1 (1:200), fibronectin (1:250), Bax (1:500), Bcl-2 (1:200) (all from Santa Cruz Biotechnology, Dallas, TX, USA), and GAPDH (1:10,000; Millipore, Billerica, MA, USA).

\section{Immunofluorescence}

Tissue immunofluorescence staining for ALK5 (1:100) and $\beta$-catenin (1:100) was performed following previously described methods [7].

\section{Real-Time qRT-PCR for Lung Cytokine Assay}

Tissue RNA extraction and q-RT-PCR were performed according to previously described methods [5]. RT-PCR primers used included IL-6: F 5'-CTTCCTACCCCAACTTCCAA-3' ${ }^{\prime}$ and R $5^{\prime}$-ACCACAGTGAGGAATGTCCA-3' (191 bp) and IFN- $\gamma$ : F $5^{\prime}$-CCAAGTTCGAGGTGAACAAC-3'; R 5'-ACTCCTTTTCCGCTTCCT-3' (110 bp). The normalization control was $18 \mathrm{~S}$ ribosomal RNA. The relative fold-change for each gene was calculated using the $\triangle \Delta$ CT method.

Choline Incorporation in Saturated Phosphatidylcholine Assay

$\left[{ }^{3} \mathrm{H}\right]$ choline (NEN Dupont, Boston, MA, USA) incorporation into saturated phosphatidylcholine, which is the major surfaceactive lipid substrate of surfactant, was determined in cultured lung explants following our previously described protocol [12].

\section{Triglyceride Uptake Assay}

$\left[{ }^{3} \mathrm{H}\right]$ triolein (PerkinElmer, Boston, MA, USA) uptake, a key marker of alveolar lipofibroblast (LIF) function, was used to quantitate triglyceride uptake in fetal rat lung explants using our previously described protocol [12].

\section{Statistical Analysis}

The Student $t$ test and ANOVA, in combination with the Tukey post hoc test, as appropriate, were used to detect group differences. The number of samples for each analysis was 4-6, from 3 separate sets of experiments. Results are expressed as means \pm SEM.

\section{Results}

We first determined whether combining PGZ with B-YL surfactant affects B-YL's bioactivity, by measuring its surface activity with captive bubble surfactometry $[8$, $10,13]$ in the presence and absence of PGZ (B-YL [100 $\mathrm{mg} / \mathrm{kg}]$ and PGZ $[1 \mathrm{mg} / \mathrm{kg}])$. We then compared this with a clinical surfactant (Infasurf) as a positive control and lipids only (DPPC:POPC: POPG 5:3:2 wt:wt:wt) as a negative control. Clinical surfactant contains both SP-B and $\mathrm{SP}-\mathrm{C}$, in contrast to B-YL that only contains a highly active SP-B peptide mimic. Minimum surface tension values of B-YL \pm PGZ were similar to those of Infasurf (Fig. 2).

Based on the ampholytic properties, the addition of $\mathrm{B}$-YL to a prostaglandin agonist is not expected to interfere with PGZ's PPAR- $\gamma$ agonist activity [14]. Therefore, 

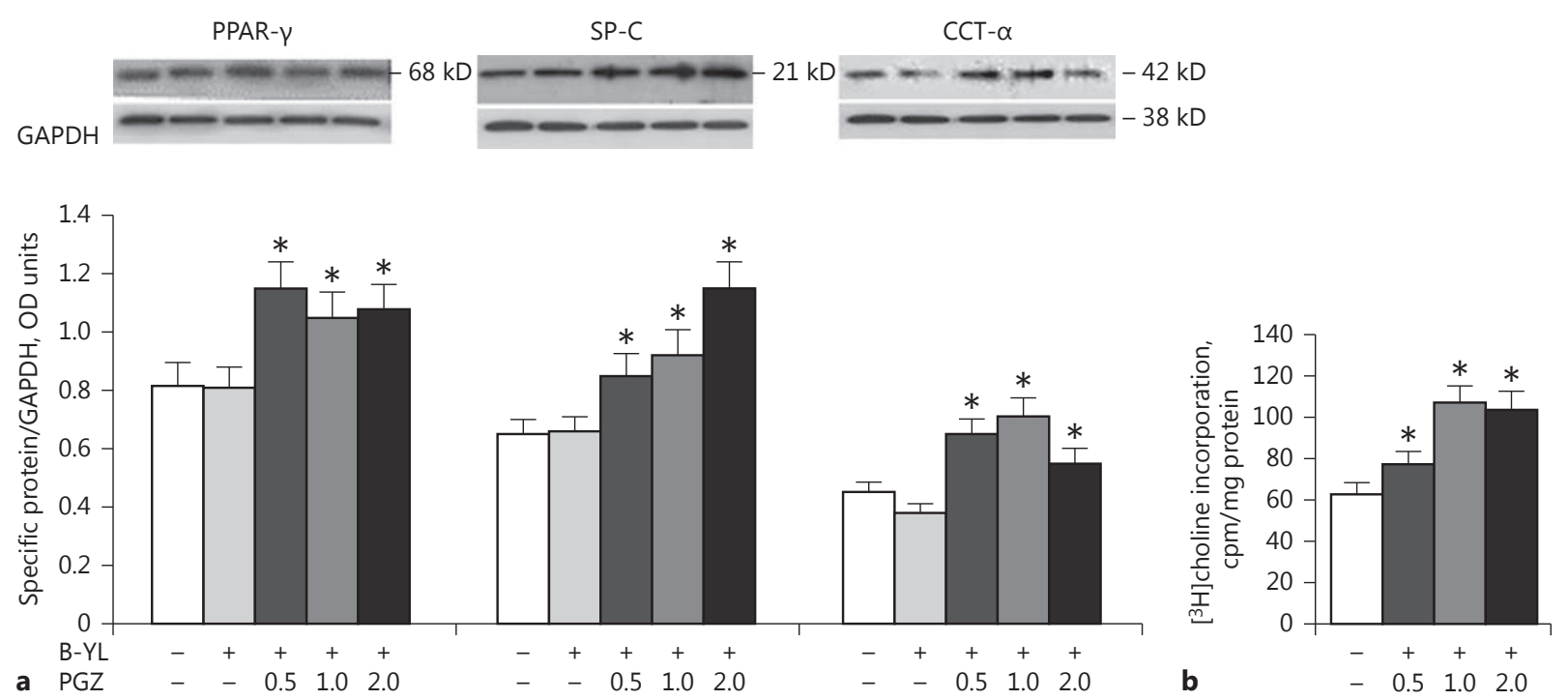

Fig. 3. Effect of B-YL on the activity of the PPAR- $\gamma$ agonist, PGZ. Embryonic day 19 fetal rat lung explants, cultured for $24 \mathrm{~h}$, under either a control condition (without B-YL or PGZ supplementation) or treated with B-YL (100 mg/kg BW) only or with PGZ $(0.5$, 1 , or $2 \mathrm{mg} / \mathrm{kg} \mathrm{BW})+\mathrm{B}-\mathrm{YL}(100 \mathrm{mg} / \mathrm{kg} \mathrm{BW})$, demonstrated sig-

we next confirmed that the addition of B-YL to PGZ did not interfere with its PPAR- $\gamma$ agonist activity, and, hence its lung maturation effect. Embryonic day 19 fetal rat lung explants were cultured for $24 \mathrm{~h}$ with or without B-YL (100 $\mathrm{mg} / \mathrm{kg}$ fetal BW) alone or with PGZ $(0.5,1$, or $2 \mathrm{mg} / \mathrm{kg}$ BW). Following this, PPAR- $\gamma$, SP-C, and CCT- $\alpha$ protein levels, along with choline incorporation into disaturated phosphatidylcholine (DSPC), all markers of lung maturation, were determined. Explants treated with PGZ + B-YL demonstrated the expected increases in PPAR- $\gamma$, SP-C, and CCT- $\alpha$ protein levels (Fig. 3a), and an increase in choline incorporation into DSPC (Fig. 3b), indicating that combining B-YL with PGZ does not affect PGZ's lung maturation activity.

Having confirmed that combining B-YL and PGZ does not interfere with surface tension reduction and lung maturational activity (of B-YL and PGZ, respectively) under in vitro conditions, we next determined if this combined approach prevents hyperoxia-induced neonatal lung injury under in vivo conditions. Postnatal day 1 rat pups were exposed to either normoxia or hyperoxia for up to $72 \mathrm{~h}$. Hyperoxia-induced decreases in PPAR- $\gamma$, SP-C, and CCT- $\alpha$ and increases in LEF- 1 and fibronectin protein levels were blocked by the concomitant administration of either PGZ or PGZ + B-YL (Fig. 4a-e). This was nificant increases in PPAR- $\gamma$, SP-C, and CCT- $\alpha$ protein levels as determined by Western blot analysis (a), along with a significant increase in $\left[{ }^{3} \mathrm{H}\right]$ choline incorporation into disaturated phosphatidylcholine (b) ${ }^{*} p<0.05$ vs. control; $\left.n=3\right)$. cpm, counts per minute.

true also for the hyperoxia-induced decrease in triolein uptake (Fig. 4f). As demonstrated by the effects on the protein levels of SP-C and CCT- $\alpha$, and triolein uptake, PGZ + B-YL administration had a more robust effect. In contrast, B-YL administration alone had no effect on any of these parameters.

Blockage of hyperoxia-induced neonatal lung injury with combined PGZ + B-YL nebulization was also shown by the normalization of hyperoxia-induced alterations in the BALF cell count, the protein content, the whole-lung apoptosis marker (the $\mathrm{Bcl}-2 / \mathrm{Bax}$ ratio), and the lung inflammatory markers IL-6 and IFN- $\gamma$ (Fig. 5). Interestingly, even though B-YL administration alone did not have any effect on most of the molecular markers of lung maturation and injury repair, it did suppress the hyperoxiainduced increase in IL- 6 and IFN- $\gamma$ expression. Lastly, since hyperoxia-induced neonatal lung injury has previously been shown to be mediated by the activation of TGF- $\beta$ and Wnt pathways, lung sections from the control, hyperoxia, and hyperoxia + PGZ + B-YL groups were stained for ALK5 and $\beta$-catenin, 2 key intermediates of TGF- $\beta$ and Wnt pathway activations, respectively. In line with the data on other markers of lung injury, a hyperoxia-induced increase in ALK5 and $\beta$-catenin staining was blocked in the PGZ + B-YL group (Fig. 6). 


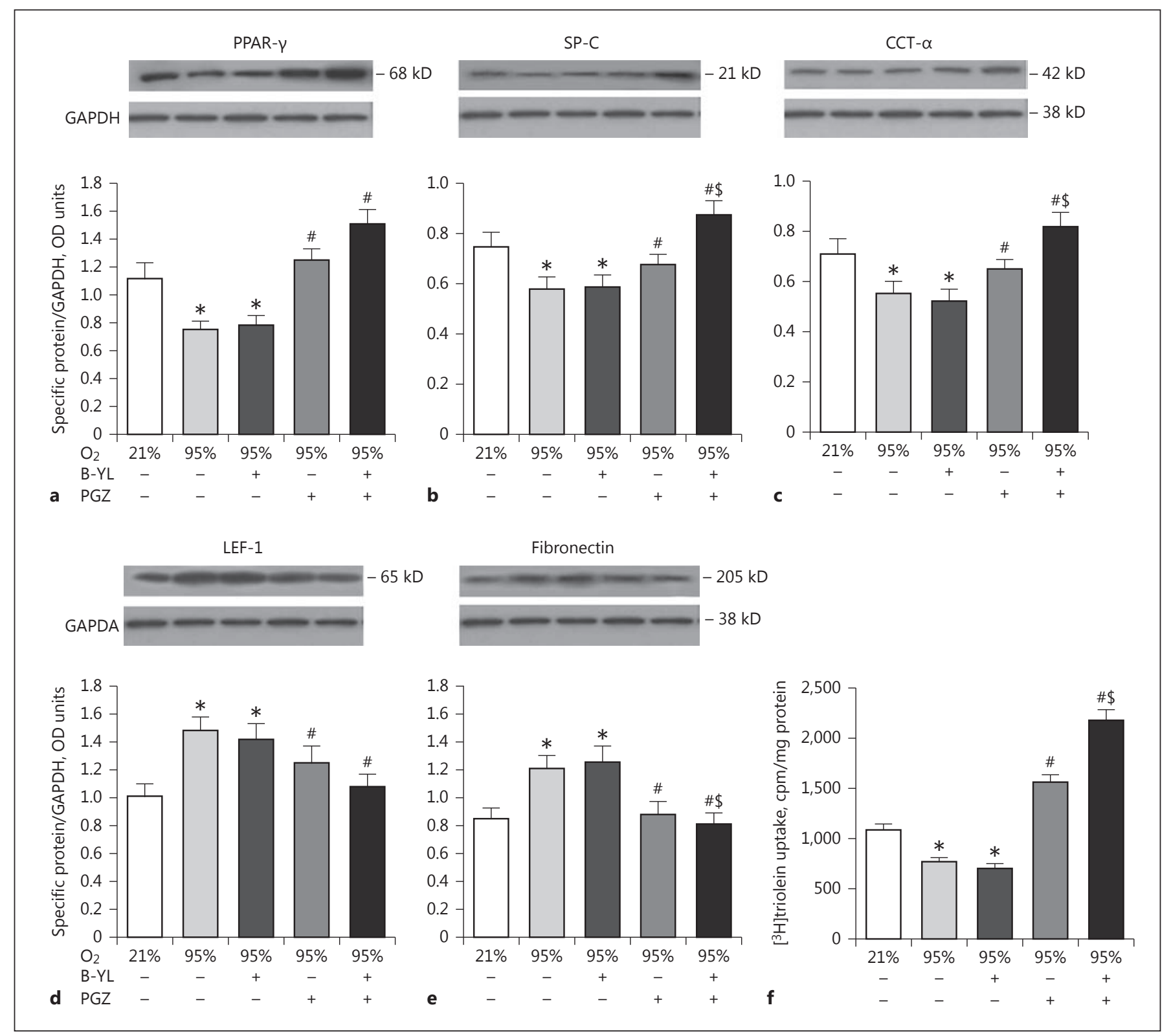

Fig. 4. Nebulized PGZ + B-YL blocks hyperoxia-induced alterations in selective markers of neonatal lung maturation more effectively than either modality alone. Postnatal day 1 rat pups were exposed to hyperoxia $\pm \mathrm{B}-\mathrm{YL}, \mathrm{PGZ}$, or PGZ $+\mathrm{B}$-YL for $72 \mathrm{~h}$. The hyperoxia-induced decrease in PPAR- $\gamma(\mathbf{a})$, SP-C (b), CCT- $\alpha$ (c) and the increase in LEF-1 (d) and fibronectin (e) protein levels were blocked by the concomitant administration of either PGZ or
PGZ + B-YL. f This was also true for the hyperoxia-induced decrease in triolein uptake. $\mathrm{PGZ}+\mathrm{B}-\mathrm{YL}$ administration had a more robust effect, as shown by the effects on the protein levels of SP-C and CCT- $\alpha$ and the triolein uptake; however, B-YL administration alone had no effect on any of these parameters. cpm, counts per minute. ${ }^{*} p<0.05$ vs. control; ${ }^{\#} p<0.05$ vs. $95 \% \mathrm{O}_{2}$, control; ${ }^{\$} p<$ 0.05 vs. PGZ $(n=3)$.

\section{Discussion}

Using both in vitro and ex vivo studies, we found that combining PGZ with the SP-B mimic B-YL does not interfere with the activity of PGZ and the surface tension-low- 


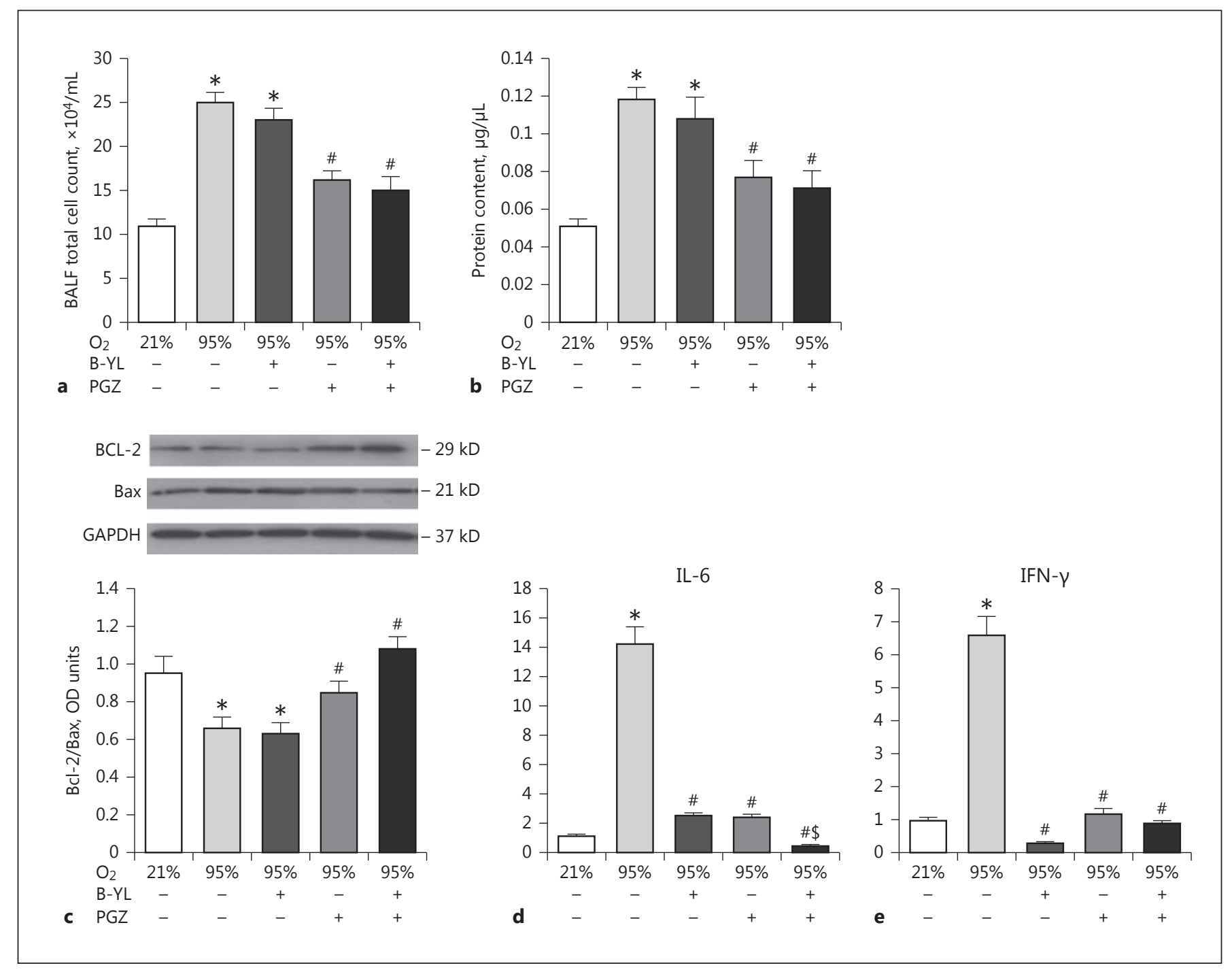

Fig. 5. Nebulized PGZ + B-YL blocks hyperoxia-induced alterations in selective markers of neonatal lung injury more effectively than either modality alone. Postnatal day 1 rat pups were exposed to hyperoxia \pm B-YL, PGZ, or PGZ + B-YL for 72 h. Hyperoxiainduced alterations in bronchoalveolar lavage fluid (BALF) total cell count (a) and protein content (b), the apoptosis marker, the Bcl-2/Bax ratio (c) and the lung inflammatory markers IL-6 (d) and IFN- $\gamma(\mathbf{e})$ were blocked by concomitant administration of either PGZ or PGZ + B-YL. ${ }^{*} p<0.05$ vs. control; ${ }^{*} p<0.05$ vs. $95 \%$ $\mathrm{O}_{2}$, control; ${ }^{\$} p<0.05$ vs. PGZ $(n=3)$. other. For example, upon hyperoxia exposure, there was relatively greater preservation of the alveolar homeostatic markers PPAR- $\gamma$, SP-C, CCT- $\alpha$, and the triolein uptake, suggesting potentially greater protection against hyperoxia-induced neonatal lung injury with the PGZ + B-YL formulation than with either component alone.

Prematurity is the underlying cause of lung immaturity leading to RDS. Various approaches including the administration of antenatal steroids, exogenous surfactant, and noninvasive and gentler modes of ventilation have shown only limited benefits in preventing RDS and BPD.
Only antenatal steroid therapy has been consistently shown to enhance lung maturation and decrease the incidence and severity of RDS. However, it is differentially more effective in females $[15,16]$, is associated with significant side effects, and does not result in a reduction in BPD. In contrast, in experimental models, the administration of PPAR- $\gamma$ agonists has been shown to reduce lung injury and enhance fetal/neonatal lung maturation safely in both males and females $[17,18]$.

One of the biggest impediments towards finding an effective preventive/therapeutic intervention against BPD 


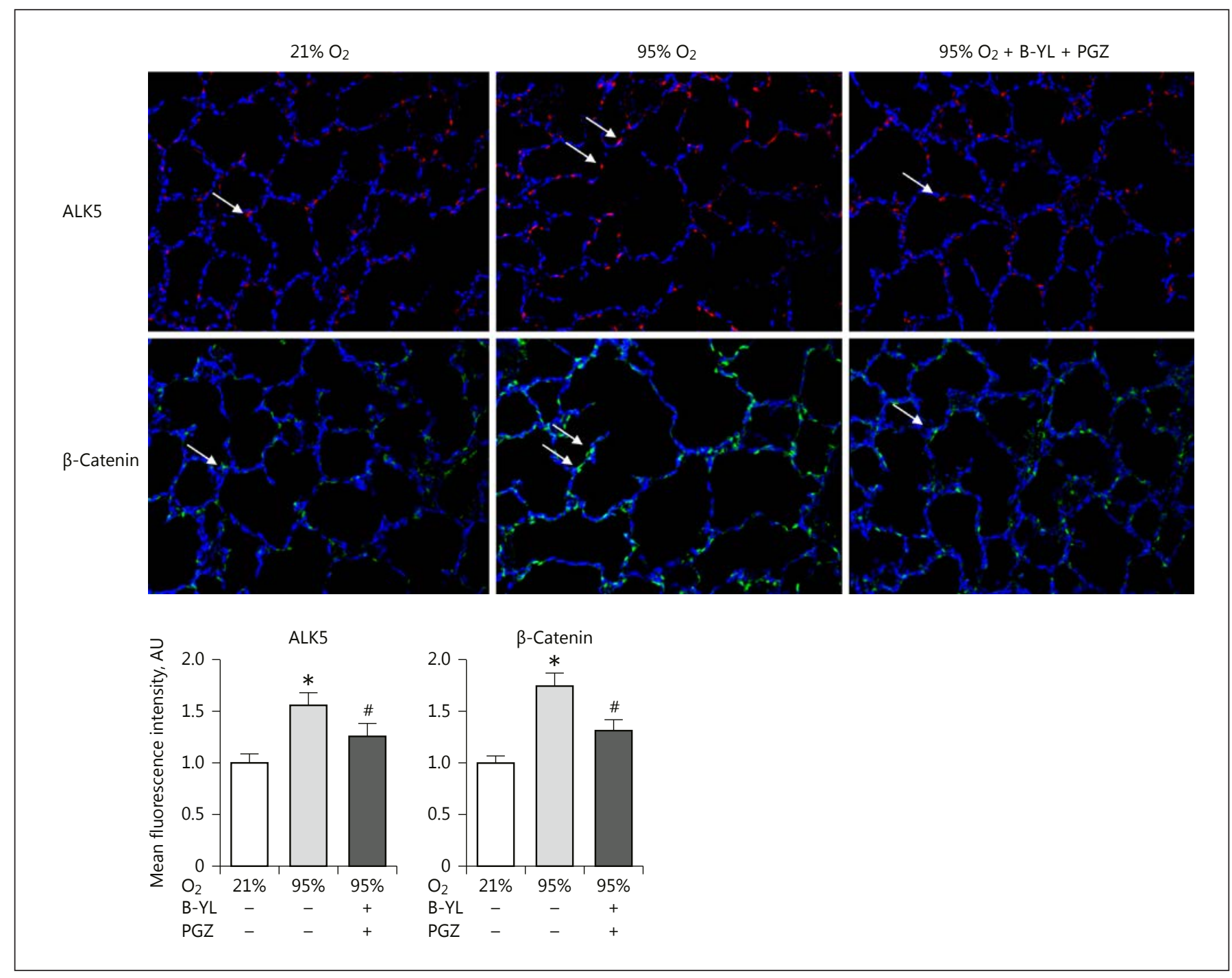

Fig. 6. Nebulized PGZ+B-YL blocks hyperoxia-induced activations of TGF- $\beta$ and Wnt signaling pathways. Postnatal day 1 rat pups were exposed to hyperoxia \pm PGZ $+\mathrm{B}$-YL for $72 \mathrm{~h}$. Hyperoxia-induced activation in TGF- $\beta$ (determined by ALK 5 protein levels, red staining) and Wnt (determined by $\beta$-catenin protein levels, green staining) signaling was blocked by concomitant PGZ + B-YL administration. Representative immunostaining pictures and relative mean fluorescence values are shown. ${ }^{*} p<0.05$ vs. control; ${ }^{\#} p<0.05$ vs. $95 \% \mathrm{O}_{2}(n=3)$. AU, arbitrary units. has been a failure to target the fundamental cellular and molecular pathways centrally involved in lung development and injury repair [3]. Combining surfactant with a PPAR- $\gamma$ agonist aims to not only reduce the manifestations of RDS acutely, but to also recapitulate the natural cellular and molecular pathways that (i) determine the normal lung structure, function, and homeostasis; (ii) are disrupted by oxotrauma, inflammation, and volutrauma; and (iii) have been shown to be normalized by PPAR- $\gamma$ agonists in multiple in vitro and in vivo models $[4-7,18]$. This approach is based on the paradigm that the develop- ing lung mesenchyme, dominated by Wnt signaling, i.e., the default pathway for muscle development, inhibits $\mathrm{C} /$ EBP- $\alpha$ and PPAR- $\gamma$, thereby actively inhibiting the adipogenic programming of the developing alveolar mesenchyme [19]. During lung morphogenesis, the mesenchymal default Wnt pathway is inhibited and the adipogenic pathway is disinhibited, resulting in the formation of lipid-laden alveolar interstitial adepithelial fibroblasts/LIFs [19]. LIFs are critical for lung homeostasis and injury repair, since they actively provide triglyceride substrate to ATII cells for surfactant synthesis, support ATII cell 
growth and differentiation, and act as an important defense against oxidative lung injury $[19,20]$. Upon exposure to volutrauma, oxotrauma, and infection, pulmonary LIFs transdifferentiate to a myogenic phenotype, myofibroblasts [18, 21]. Myofibroblasts do not support ATII cell growth or differentiation, whereas LIFs do [20], hence the critical importance of maintaining the proper balance of both, in order to direct lung development. Previous studies have demonstrated that all of the abovementioned BPD inducers cause the downregulation of alveolar LIF PPAR- $\gamma$ expression [18], and thus inhibit normal lung development, but that PPAR- $\gamma$ agonists mitigate, and, in some cases, even reverse the lung damage $[18,20-22]$. However, it is important to point out that although we have focused on the role of PPAR- $\gamma$ in LIF biology, it also exerts a prominent injury repair effect via a variety of other mechanisms [23].

Other advantages of our approach include the potential delivery of the nebulized PGZ + B-YL formulation directly to the lung, which should result in the maximum therapeutic effect, limit systemic side effects, and circumvent the issues pertaining to bioavailability and first-pass metabolism if administered orally. Furthermore, the aerosolized delivery of PGZ + B-YL eliminates intubation and associated complications. However, detailed pharmacokinetics studies are needed to determine the biodistribution of PGZ and B-YL in the lung, blood, and other organs as well as any systemic effects, before this proposed approach can be translated to the bedside.

As alluded to above, B-YL is an innovative surfactant developed specifically to resist hyperoxia-induced inacti- vation [24]. It is both stable and highly active in its native state and after the addition of PGZ during in vitro testing (Fig. 2). Moreover, it has a low viscosity, aerosolizes easily, and can be produced inexpensively, all highly relevant attributes for its translation into clinical practice. Since the PGZ + B-YL formulation effectively blocked lung injury, even following an extremely high $\mathrm{O}_{2}$ exposure, i.e., $95 \% \mathrm{O}_{2}$ exposure for $72 \mathrm{~h}$ (a scenario which is rarely used in contemporary neonatal practice), we are confident that it will also be effective against the usual lower levels of hyperoxia exposure frequently encountered in neonatal intensive care units. While PGZ was used as a prototype PPAR- $\gamma$ agonist in this study, other PPAR- $\gamma$ agonists might prove to be safer and even more effective. It is also worth noting that the lung maturational effect of PPAR- $\gamma$ agonists is not restricted to PGZ, but has also been seen with curcumin, troglitazone, rosiglitazone, and prostaglandin $\mathrm{J}_{2}[4-7,18,21,25]$.

\section{Disclosure Statement}

The authors declare no conflicts of interest.

\section{Funding Sources}

We received grant support from the NIH (HD51857, HL107118, HD071731, and HL127237), TRDRP (17RT-0170, and 23RT0018), and the Bill and Melinda Gates Foundation (OPP1112090).

\section{References}

1 Maitre NL, Ballard RA, Ellenberg JH, Davis SD, Greenberg JM, Hamvas A, Pryhuber GS; Prematurity and Respiratory Outcomes Program: Respiratory consequences of prematurity: evolution of a diagnosis and development of a comprehensive approach. J Perinatol 2015;35:313-321.

2 Martin RJ, Fanaroff AA: The preterm lung and airway: past, present, and future. Pediatr Neonatol 2013;54:228-234.

-3 Silva DM, Nardiello C, Pozarska A, Morty RE: Recent advances in the mechanisms of lung alveolarization and the pathogenesis of bronchopulmonary dysplasia. Am J Physiol Lung Cell Mol Physiol 2015;309:L1239-L1272.

4 Wang Y, Santos J, Sakurai R, Shin E, Cerny L, Torday JS, Rehan VK: Peroxisome proliferator-activated receptor gamma agonists enhance lung maturation in a neonatal rat model. Pediatr Res 2009;65:150-155.
Morales E, Sakurai R, Husain S, Paek D, Gong M, Ibe B, Li Y, Husain M, Torday JS, Rehan VK: Nebulized PPAR $\gamma$ agonists: a novel approach to augment neonatal lung maturation and injury repair in rats. Pediatr Res 2014;75: 631-640.

-6 Dasgupta C, Sakurai R, Wang Y, Guo P, Ambalavanan N, Torday JS, Rehan VK: Hyperoxia-induced neonatal rat lung injury involves activation of TGF- $\beta$ and Wnt signaling and is protected by rosiglitazone. Am J Physiol Lung Cell Mol Physiol 2009;296:L1031-L1041.

7 Sakurai R, Villarreal P, Husain S, Liu J, Sakurai T, Tou E, Torday JS, Rehan VK: Curcumin protects the developing lung against longterm hyperoxic injury. Am J Physiol Lung Cell Mol Physiol 2013;305:L301-L311.
8 Walther FJ, Waring AJ, Hernandez-Juviel JM, Gordon LM, Wang Z, Jung CL, Ruchala R, Clark AP, Smith WM, Sharma S, Notter RH: Critical structural and functional roles for the $\mathrm{N}$-terminal insertion sequence in surfactant protein B analogs. PLoS One 2010;5:e8672.

-9 Walther FJ, Hernandez-Juviel JM, Waring AJ: Aerosol delivery of synthetic lung surfactant. PeerJ 2014;2:e403.

10 Dubus JC, Vecellio L, De Monte M, Fink JB, Grimbert D, Montharu J, Valat C, Behan N, Diot P: Aerosol deposition in neonatal ventilation. Pediatr Res 2005;58:10-14.

-11 Finer NN, Merritt TA, Bernstein G, Job L, Mazela J, Segal R: An open label, pilot study of Aerosurf ${ }^{\mathbb{B}}$ combined with nCPAP to prevent RDS in preterm neonates. J Aerosol Med Pulm Drug Deliv 2010;23:303-309.
Pioglitazone and SP-B Can Prevent Neonatal Lung Injury 
12 Rehan VK, Wang Y, Sugano S, Santos J, Patel S, Sakurai R, Boros LG, Lee WP, Torday JS: In utero nicotine exposure alters fetal rat lung alveolar type II cell proliferation, differentiation, and metabolism. Am J Physiol Lung Cell Mol Physiol 2007;292:L323-L323.

13 Walther FJ, Waring AJ, Hernandez-Juviel JM, Gordon LM, Schwan AL, Jung C-L, Chang Y, Wang Z, Notter RH: Dynamic surface activity of a fully synthetic phospholipase-resistant lipid/peptide lung surfactant. PLoS One 2007; 2:e1039.

14 Giaginis C, Theocharis S, Tsantili-Kakoulidou A: Investigation of the lipophilic behaviour of some thiazolidinediones. Relationships with PPAR- $\gamma$ activity. J Chromatogr B Analyt Technol Biomed Life Sci 2007;857: 181-187.

15 Effect of antenatal dexamethasone administration on the prevention of respiratory distress syndrome. Am J Obstet Gynecol 1981; 141:276-287.
16 Papageorgiou AN, Colle E, Farri-Kostopoulos E, Gelfand MM: Incidence of respiratory distress syndrome following antenatal betamethasone: role of sex, type of delivery, and prolonged rupture of membranes. Pediatrics 1981;67:614-617.

17 Lee C, Sakurai R, Shen H, Rehan V: Antenatal PPAR- $\gamma$ pioglitazone stimulates fetal lung maturation equally in males and females. Abstract \#53, 2017 Western Medical Research Conference, Jan 26-28, Carmel, CA, USA.

18 Torday JS, Rehan VK: Developmental cell/ molecular biologic approach to the etiology and treatment of bronchopulmonary dysplasia. Pediatr Res 2007;62:2-7.

19 Torday JS, Rehan VK: Up-regulation of fetal rat lung parathyroid hormone-related protein gene regulatory network down-regulates the Sonic Hedgehog/Wnt/ $\beta$-catenin gene regulatory network. Pediatr Res 2006;60:382-388.

20 Torday JS, Torres E, Rehan VK: The role of fibroblast transdifferentiation in lung epithelial cell proliferation, differentiation, and repair in vitro. Pediatr Pathol Mol Med 2003;22: 189-207.
21 Rehan VK, Torday JS: The lung alveolar lipofibroblast: an evolutionary strategy against neonatal hyperoxic lung injury. Antioxid Redox Signal 2014;21:1893-1904.

22 Rehan VK, Sakurai R, Wang Y, Santos J, Huynh K, Torday JS: Reversal of nicotine-induced alveolar lipofibroblast-to-myofibroblast transdifferentiation by stimulants of parathyroid hormone-related protein signaling. Lung 2007;185:151-159.

23 Reddy RC, Rehan VK, Roman J, Sime PJ: PPARs: Regulators and translational targets in the lung. PPAR Res 2012;2012:342924.

24 Walther FJ, Gordon LM, Waring AJ: Design of surfactant protein B peptide mimics based on the saposin fold for synthetic lung surfactants. Biomed Hub 2016;1:451076.

25 Sakurai R, Li Y, Torday JS, Rehan VK: Curcumin augments lung maturation, preventing neonatal lung injury by inhibiting TGF- $\beta$ signaling. Am J Physiol Lung Cell Mol Physiol 2011;301:L721-L730.

26 http://zhanglab.ccmb.med.umich.edu/ITASSER. 